\title{
A rare case of Brugada syndrome induced by hyperglycemia
}

\author{
Brandon W. Knopp", Bailey Pierce ${ }^{1}$, Vishnu Muppala², Jonathan Rosman ${ }^{3}$, Jeniel \\ Parmar ${ }^{*}, 3$ \\ ${ }^{1}$ Charles E. Schmidt College of Medicine, Florida Atlantic University, Boca Raton, FL, USA; \\ ${ }^{2}$ Maimonides Medical Center, FL, USA; ${ }^{3}$ Boca Raton Regional Hospital, Boca Raton, FL, USA
}

\begin{abstract}
Brugada syndrome is a rare genetic disorder of the cardiac sodium channels associated with an increased risk of sudden cardiac death. It is characterized by an electrocardiogram (EKG) showing a right bundle branch block with an elevation in the ST segment. This condition is associated with mutations in several pathologic genes including the most notable mutation in the SCN5A gene, which encodes for a voltage-gated cardiac sodium channel. The Brugada pattern on EKG can be spontaneous but can also be induced by a variety of etiologies including fever, electrolyte abnormalities, increased vagal tone and drugs such as sodium channel blockers, calcium channel blockers, tricyclic antidepressants and alcohol. One uncommon cause of Brugada syndrome is hyperglycemia. Of particular importance in diabetic patients, hyperglycemia can induce chronic cardiovascular complications as well as acute cardiac events via the induction of the Brugada pattern on EKG. We present a case of a 21-year-old non-insulin compliant diabetic man presenting to the Emergency Department with diabetic ketoacidosis (DKA) who exhibits the Brugada pattern EKG prior to developing ventricular tachycardia followed by cardiac arrest. The patient's condition was induced by prolonged hyperglycemia in the setting of DKA with relatively mild electrolyte and $\mathrm{pH}$ abnormalities. Herein, this case is presented to highlight the Brugada pattern leading to cardiac arrest as a potential consequence of hyperglycemia and inform physicians on its incidence.
\end{abstract}

Keywords: Brugada syndrome; sudden cardiac death; hyperglycemia

\section{Introduction}

Brugada syndrome is a rare genetic disorder of the cardiac sodium channels associated with an increased risk of sudden cardiac death. It is characterized by an electrocardiographic (EKG) right bundle branch block (RBBB) and ST-segment elevation [1]. Symptoms which present in Brugada syndrome may include fainting, palpitations, seizures and cardiac arrest [2].

Received: May 2021; Accepted after review: July 2021; Published: July 2021.

*Corresponding author: Jeniel Parmar, Boca Raton Regional Hospital, 800 Meadows Rd, Boca Raton, FL 33486, USA

Email: jenielparmar@gmail.com
Patients exhibiting the Brugada pattern on EKG are also at a higher risk of ventricular tachycardia and ventricular fibrillation, potentially leading to sudden cardiac death [1]. This condition is associated with mutations in several pathologic genes including the most notable mutation in the SCN5A gene, which encodes for a voltage-gated cardiac sodium channel [3]. Brugada Syndrome has also been associated with diabetic ketoacidosis (DKA) and the subsequent electrolyte and $\mathrm{pH}$ irregularities. In rare cases, Brugada Syndrome has been induced by prolonged hyperglycemia in the context of DKA [4]. The Brugada pattern on EKG can resolve upon correction of the underlying cause or precede ventricular arrhythmias and cardiac arrest [2]. Our patient, with a likely genetic predisposition 
to Brugada syndrome and insulin noncompliance, went into sudden cardiac arrest in the Emergency Department (ED) from hyperglycemia. This case offers insight into the management of Brugada syndrome induced by hyperglycemia and highlights cardiac arrest as a rare consequence of prolonged hyperglycemia in patients with Brugada syndrome.

\section{Case report}

A 21-year-old man with a past medical history of type I diabetes on insulin presented to the ED with a 1-day history of syncope and vomiting associated with generalized weakness, abdominal pain and dry mouth. The patient denied fever, chills, chest pain, shortness of breath, headache, diaphoresis, hematemesis and back pain. The patient stated his insulin pump had not been working for the past several days and his home blood sugar measured in the 500 s the day before. His past medical history was significant for poor adherence to insulin and several episodes of DKA. He also mentioned multiple syncopal episodes in past without any prodromal symptoms. He described multiple family members with sudden cardiac death at an early age.

\section{Physical examination}

The patient was alert and oriented to person, place and time upon arrival to the ED. He was afebrile $\left(36.5^{\circ} \mathrm{C}\right)$, hypotensive $(97 / 39)$, tachycardic (117 beats per minute) and tachypneic (23 respirations per minute). Cardiac exam showed tachycardia at normal rhythm with normal S1 and S2 and no murmur or rub. Pulses in the extremities were intact. Abdominal exam showed a soft, nondistended abdomen with mild tenderness. Skin was warm and dry with no cyanosis.

\section{Laboratory results and EKG}

Laboratory results taken upon admission were significant for hyperkalemia $(6.0 \mathrm{mmol} / \mathrm{L})$, hyponatremia $(122 \mathrm{mmol} / \mathrm{L})$, hypochloremia $(85 \mathrm{mmol} / \mathrm{L})$, and hypercapnia $(7 \mathrm{mmol} / \mathrm{L})$. Labs also showed a high anion gap metabolic acidosis with compensated respiratory alkalosis. Ten minutes after arrival, his blood glucose measured critically high $(742 \mathrm{mg} / \mathrm{dL})$ with a blood $\mathrm{pH}$ of 7.11 . His renal function tests were also abnormal with a low glomerular filtration rate (GFR) $\left(45 \mathrm{~mL} / \mathrm{min} / 1.73 \mathrm{~m}^{2}\right)$, high blood urea nitrogn $(24 \mathrm{mg} / \mathrm{dL})$ and high creatinine $(2.0 \mathrm{mg} / \mathrm{dL})$. Three hours after arrival, his urine was positive for glucosuria, proteinuria, hematuria, and ketonuria.

An EKG taken 30 minutes after arrival (Figure 1) showed incomplete right bundle branch block. A follow up EKG taken about 2 hours after arrival (Figure 2) showed sinus tachycardia with a normal rhythm and an IV conduction block replacing the incomplete RBBB seen in the previous EKG. The second EKG also showed hyperacute appearing $T$ waves and pattern in leads $\mathrm{V} 1-\mathrm{V} 2$ consistent with Brugada pattern type I. A third EKG (Figure 3) was taken almost 3 hours after arrival to the ED which showed a wide QRS tachycardia with premature ventricular contractions (PVCs) and resolution of Brugada pattern type I. At this time, insulin was still unable to be given due to a second blood sampling being hemolyzed resulting in the absence of an accurate potassium reading. Just after the $3^{\text {rd }}$ EKG was taken, the patient developed ventricular tachycardia followed by a cardiac arrest.

\section{Diagnosis and treatment}

Based on the evidence of the clinical findings, laboratory, and EKG results the patient was diagnosed with diabetic ketoacidosis (DKA) inducing Brugada pattern type I and subsequent ventricular tachycardia followed by cardiac arrest. CPR was initiated and endotracheal intubation was performed under emergent conditions without complications. Compressions were started just after the pulse was lost and ACLS was followed, with subsequent return of spontaneous circulation. In addition to insulin, he was treated with electrolytes including $1 \mathrm{~g}$ calcium gluconate and sodium chloride $(0.9 \%$ drip, $1,000 \mathrm{~mL}$ via IV) to balance his abnormal levels. 


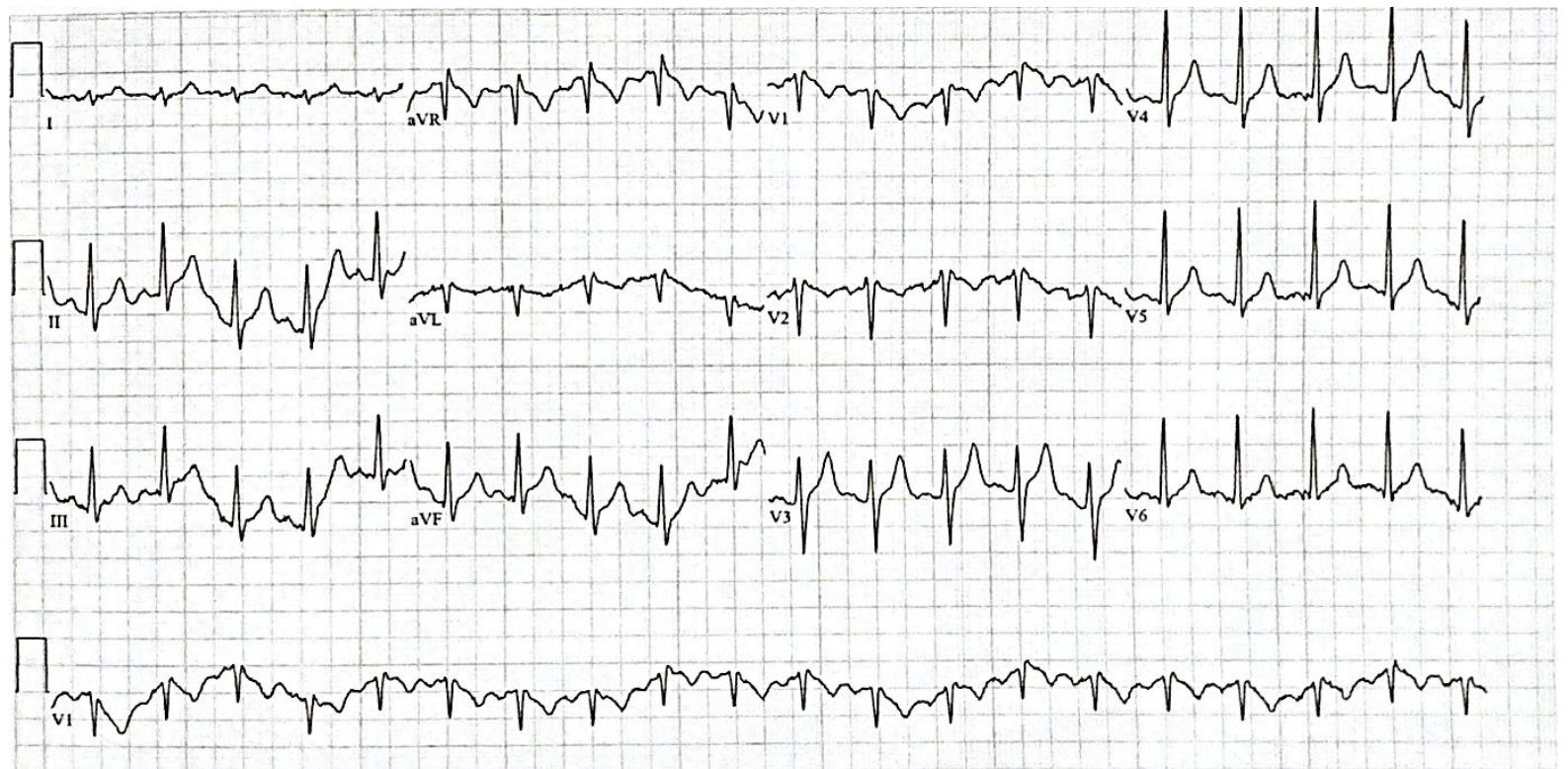

Fig. 1. Sinus tachycardia, incomplete right bundle branch block with minimal ST elevation in V1 and V2 (standard position); QT/QTc = 344/483.

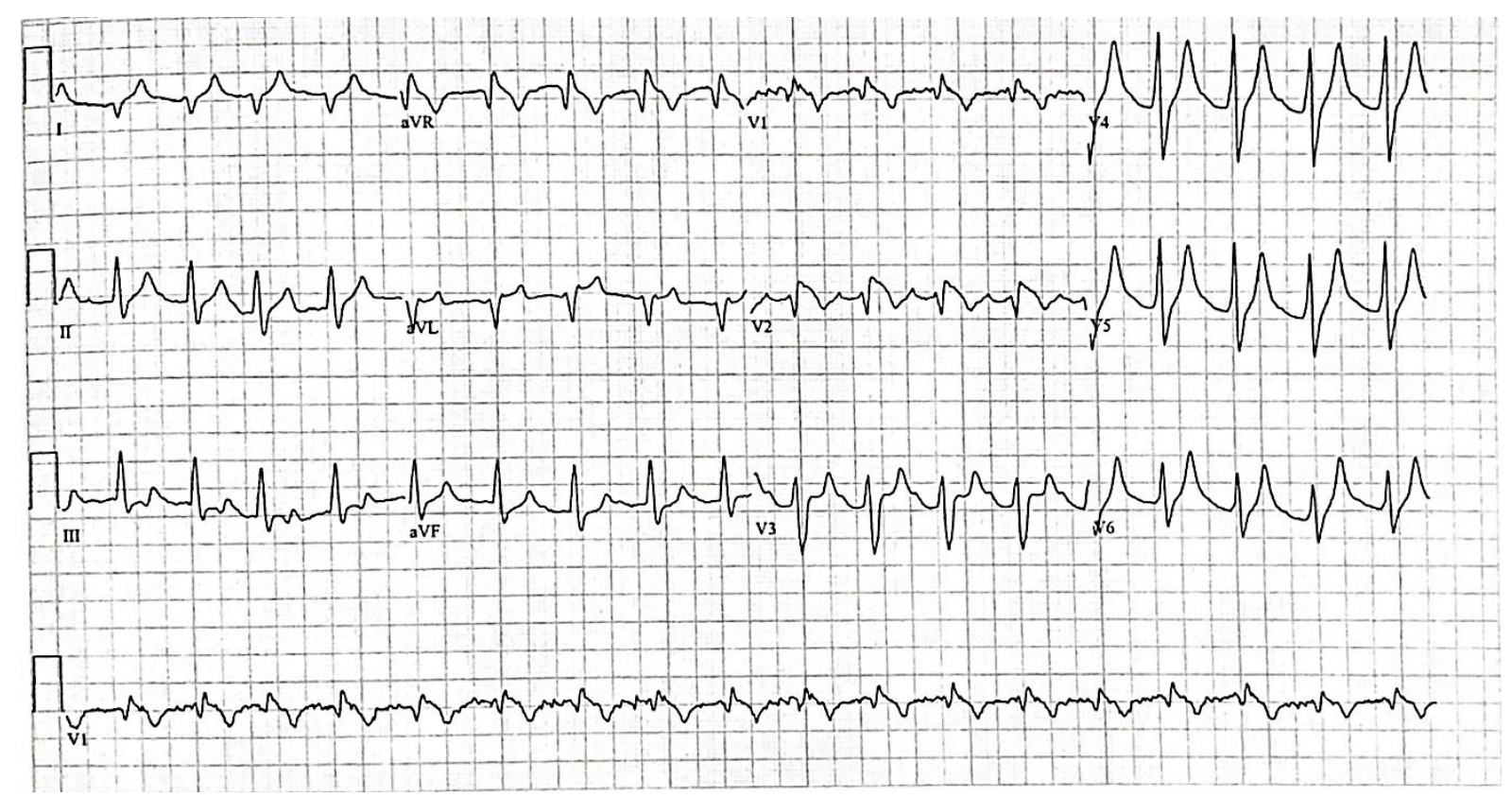

Fig. 2. Sinus tachycardia, first degree AV block, incomplete right bundle branch block with ST elevation in V1 and V2 (standard position) consistent with Brugada Pattern Type I. 


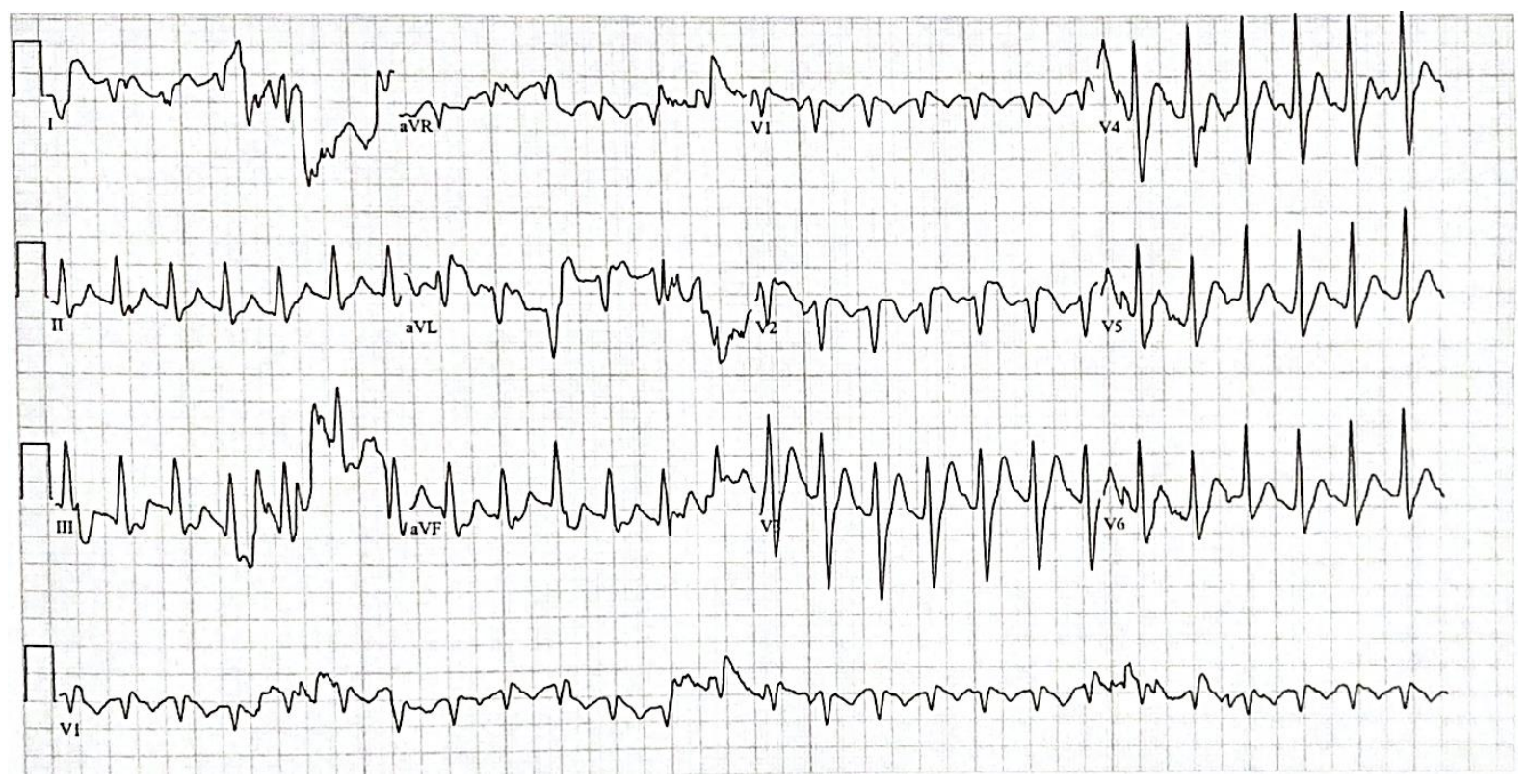

Fig. 3. Sinus tachycardia with Type III Brugada pattern characterized by $<2 \mathrm{~mm}$ ST elevation in V1 and V2 (standard position); QT/QTC = 294/473.

\section{Discussions}

Brugada syndrome is a rare and potentially fatal cardiac arrhythmia leading to up to $20 \%$ of all recorded sudden death events in individuals with structurally normal hearts [5]. Brugada syndrome is characterized by a ventricular arrhythmia, which can be asymptomatic or lead to cardiac arrest, syncope or sudden cardiac death. Accounting for up to $12 \%$ of all sudden cardiac deaths according to some sources, Brugada syndrome may lead to up to 39,000 adult deaths in the United States each year with an average age of incidence of 40 years old [5, $6]$.

In a scoping review conducted by Haseeb et al., 22 cases of Brugada syndrome were examined in individuals who initially presented with DKA. While these reports often cite electrolyte abnormalities as the inciting factor of Brugada syndrome, they rarely discuss the role of hyperglycemia [7]. We postulate that hyperglycemia itself may be an inducing agent, even in the setting of relatively normal electrolytes. The first indication of this connection between blood glucose and cardiac events in Brugada syndrome is a report from the Thai ministry of Public Health in 1990. They found an association between Brugada syndrome patients consuming a large carbohydrate-heavy meal the night they passed away in their sleep [8]. This association was further investigated in Nogami et al. who found the accentuation of STsegment in high-risk Brugada syndrome patients following infusion of glucose or glucose and insulin solution, even with little to no isoelectric separation between the Brugada syndrome patients and controls [9]. Among the cases described by Haseeb et al. in their scoping review, only 3 of the 22 cases included between 1978 and 2018 were comparable to our case in terms of a patient in DKA with relatively mild electrolyte abnormalities and acidosis, but also severe hyperglycemia inducing Brugada syndrome [7]. The type I diabetic patient described in this case provides an interesting addition to the discussion as hyperglycemia preempted the onset of Brugada pattern on EKG. Our patient is unique in that unlike many other cases of diabetics in DKA who developed Brugada syndrome, his electrolyte abnormalities were relatively insignificant, pointing to hyperglycemia as the primary contributor to development of the Brugada pattern. He was also acidotic with a $\mathrm{pH}$ of 7.12 , which is low but like the electrolytes, not severe enough to be the primary inciting factor.

Patients with Brugada Syndrome are clearly more sensitive to fluctuations in internal 
conditions than non-affected patients, accounting for the potentially fatal outcomes following large changes in blood glucose and/or insulin secretion [9]. The trophic effects of insulin are well-known, with effects ranging from the lowering of blood glucose to modulation of $\mathrm{Na}^{+}$and $\mathrm{K}^{+}$pump currents in cardiac tissue, potentially contributing to an inward current which may reduce ST-segment elevation. While hyperglycemia was the main inciting agent for Brugada syndrome in our patient, available evidence indicates that the Brugada pattern resolves with correction of the underlying cause [2]. As a result, the primary treatments for our patient would have been IV fluids and insulin. However, as mentioned in the case presentation, our patient was unable to be given insulin due to an inability to obtain an accurate potassium measurement following the initial lab study taken upon admission. The absence of a potassium level prevented insulin administration because the potential risk of hypokalemia is far more common than the development of Brugada syndrome. Hypokalemia occurs in up to $21 \%$ of hospitalized patients and $2 \%$ to $3 \%$ of outpatients and is accompanied by an increase in cardiovascular mortality and heart failure mortality by up to 10 -fold [10].

Hypokalemia is an established risk for cardiac events and significant clinical measures are taken to prevent this condition, namely the continued monitoring of serum potassium. However, hyperglycemia-induced Brugada syndrome, while rare, can lead to dangerous cardiac complications as well. Due

\section{References}

1. Brugada $P$, Brugada J. Right bundle branch block, persistent ST segment elevation and sudden cardiac death: a distinct clinical and electrocardiographic syndrome. A multicenter report. J Am Coll Cardiol 1992; 20(6):13911396. doi: 10.1016/0735-1097(92)90253-j

2. Sarquella-Brugada G, Campuzano O, Arbelo E, Brugada J, Brugada R. Brugada syndrome: clinical and genetic findings. Genet Med 2016; 18(1):3-12. doi:10.1038/gim.2015.35

3. Chen $Q$, Kirsch GE, Zhang D, et al. Genetic basis and molecular mechanism for idiopathic ventricular fibrillation. Nature 1998; 392(6673):293-296. doi: 10.1038/32675 to its potentially fatal outcomes, we propose additional clinical consideration for Brugada syndrome in the treatment of hyperglycemic patients. This association between hyperglycemia and Brugada syndrome is especially important in diabetic patients and may contribute in part to the greater risk of cardiac events in diabetic patients. Our case is an example of the dangers of hyperglycemiainduced Brugada syndrome and serves to better inform the care of patients with hyperglycemia to avoid this potential fatal cardiac condition.

\section{Conclusions}

Brugada syndrome is a rare condition; however, its development should still be monitored in DKA patients as it can alert the physician to the greater potential for sudden cardiac arrest in patients of all ages. The case presented above is an example of how prolonged hyperglycemia can lead to cardiac arrest by inducing Brugada Syndrome in otherwise healthy individuals. This report serves to bring attention to this important association and inform physicians on the potential development of Brugada Syndrome leading to cardiac arrest induced by hyperglycemia.

\section{Competing interests}

The authors declare that they have no competing interests.
4. Kovacic JC, Kuchar DL. Brugada pattern electrocardiographic changes associated with profound electrolyte disturbance. Pacing Clin Electrophysiol 2004; 27(7):1020-1023. doi: 10.1111/j.1540-8159.2004.00579.x

5. Brugada R, Campuzano $O$, Sarquella-Brugada G, Brugada J, Brugada P. Brugada syndrome. Methodist Debakey Cardiovasc J 2014; 10(1):25-28. doi:10.14797/mdcj-10-1-25

6. Sudden Cardiac Arrest Foundation. Sudden cardiac arrest: a healthcare crisis. 2017. [https://www.sca-aware.org/about-sca available at $07 / 22 / 2021]$ 
7. Chanavirut $R$, Leelayuwat N. Brugada syndrome and carbohydrate metabolism. J Cardiol Curr Res 2017; 8(5):00294. doi:10.15406/jccr.2017.08.00294

8. Haseeb S, Kariyanna PT, Jayarangaiah A, et al. Brugada pattern in diabetic ketoacidosis: a case report and scoping study. Am J Med Case Rep 2018; 6(9):173-179. doi:10.12691/ajmcr6-9-2
9. Nogami A, Nakao M, Kubota $S$, et al. Enhancement of J-ST-segment elevation by the glucose and insulin test in Brugada syndrome. Pacing Clin Electrophysiol 2003; 26(1):332-337. doi:10.1046/j.14609592.2003.00044.x.

10. Viera A, Wouk N. Potassium disorders: hypokalemia and hyperkalemia. Am Fam Physician 2015; 92(6):487-495. www.aafp.org/afp/2015/0915/p487.html. 